\title{
El papel de la Universidad en la Cooperación Internacional al Desarrollo. Los ODS y el horizonte 2030
}

\author{
Cano-Ginés, Antonio \\ Universidad de La Laguna, La Laguna, España \\ acangi@ull.edu.es
}

\section{Resumen}

Hoy pocos dudan de que la Universidad ha de ser un actor importante en la Cooperación al Desarrollo. Actualmente colabora tanto en el desarrollo de estrategias y acciones conjuntas de los centros de educación superior como en las acciones de difusión de los distintos trabajos que se realizan en las universidades en materia de Cooperación. Asimismo, contribuye a la mejora de la relación con otros agentes de la cooperación con objeto de fomentar el trabajo en red, fundamental en los proyectos de cooperación internacional educativa.

En esta comunicación mostraremos cómo la cooperación universitaria al desarrollo desde la docencia, la investigación, la innovación y la gestión no solo es posible sino necesaria. Los Objetivos de Desarrollo Sostenible (ODS) abarcan todas las ramas de conocimiento y debemos ser capaces de incidir en ello.

\section{Abstract}

Today few people doubt that the University has to be an important actor in the Development Cooperation. Currently he collaborates both in the development of joint strategies and actions of the higher education centers and in the dissemination actions of the different works carried out in the universities in matters of Cooperation. It also contributes to the improvement of the relationship with other agents of cooperation in order to promote networking, which is fundamental in international educational cooperation projects.

In this communication we will show how university cooperation for development from teaching, research, innovation and management is not only possible but necessary. The Sustainable Development Goals (SDGs) cover all branches of knowledge and we must be able to influence them.

Palabras clave: Objetivos de Desarrollo Sostenible, Cooperación Universitaria, Universidad y Cooperación, Cooperación Internacional al desarrollo.

Keywords: Sustainable Development Goals, University Cooperation, University and Cooperation, International Development Cooperation.

\section{INTRODUCCIÓN}

La universidad española, aun teniendo recursos financieros y tecnológicos limitados, cuenta con el capital humano y el talento necesario en todas las ramas de conocimiento para abordar con solvencia los objetivos de desarrollo sostenible (ODS) y ser un agente colaborador de primer orden en la cooperación Internacional al desarrollo.

Visibilizar la universidad ante la sociedad como motor de colaboración y dar a conocer sus actuaciones en los diversos ámbitos en los que interviene como agente de cooperación debe ser el objetivo fundamental, pero también deben constituirse en meta primordial el análisis y la asunción de nuevos retos que favorezcan el desarrollo humano y los valores de solidaridad.

Asimismo, hay que poner en valor el hecho de que la universidad cuenta con la masa crítica necesaria y con las relaciones internacionales suficientes para afrontar desafíos globales a medio y largo plazo en campos muy distintos, especialmente en el educativo, pero no solo, y cuenta, además, con una http://doi.org/10.25145/c.educomp.2018.16.082 
alta credibilidad y neutralidad, aspectos fundamentales para afrontar retos en campos tan sensibles como la gobernanza, los derechos humanos y la propuesta y resolución de problemas relacionados con el medio ambiente, la seguridad alimentaria, las energías renovables o el consumo responsable.

El horizonte 2030 es sin duda una oportunidad para la Universidad española. La Cooperación Internacional debe considerarse un ejercicio de responsabilidad universitaria en el que toda su comunidad, Personal Docente e Investigador, Alumnado y Personal de Administración y Servicios, es imprescindible.

Tenemos algo más de una década por delante para que la necesaria sensibilización empape nuestros centros a modo de lluvia fina, y para ello es vital dar a conocer la importancia del papel que desempeña la Universidad, promoviendo en el seno de la misma el debate, y favorecer así la adquisición de compromisos con acciones y estrategias orientadas a conseguir transversalizar los ODS en todos los ámbitos de actuación posibles.

\section{LA COOPERACIÓN UNIVERSITARIA AL DESARROLLO}

\subsection{Definición de Cooperación Universitaria al Desarrollo}

La Cooperación Universitaria al Desarrollo (CUD) se entiende como un «conjunto de actividades llevadas a cabo por la comunidad universitaria y orientadas a la transformación social en los países más desfavorecidos, en pro de la paz, la equidad, el desarrollo humano y la sostenibilidad medioambiental en el mundo». Así se recoge en el artículo 9 del Código de conducta de las Universidades en materia de Cooperación al Desarrollo (CRUE, 2015).

Los antecedentes a esta definición se encuentran en la publicación de la ESCUDE (Estrategia de las Universidades españolas en materia de Cooperación al Desarrollo), en el año 2000. En ella se distingue entre cooperación internacional, cooperación al desarrollo y ayuda humanitaria, y es aquí donde se señala que «la cooperación Internacional debe ser entendida como aquella modalidad de relaciones entre países que persiguen un beneficio mutuo. La Cooperación al Desarrollo es una parte de la Cooperación Internacional que, con similar propósito, se establece entre países con distinto nivel de desarrollo, con unos fines concretos (consolidación democrática, desarrollo económico y social sostenible, lucha contra la pobreza, protección del medio ambiente, entre otros) y todo ello planteado en términos de corresponsabilidad entre las contrapartes. En ese sentido no debe confundirse con la ayuda humanitaria y, consecuentemente, debe exigir un esfuerzo de las contrapartes, aunque no puede ser el mismo en todos los países» (CRUE, 2000: 1).

Es importante señalar que a partir de la ESCUDE se han desarrollado una serie de trabajos que pretenden contribuir a perfeccionar las acciones que en materia de cooperación se llevan a cabo en las universidades españolas entre los que destacan: Universidad: Compromiso social y voluntariado (2001), Protocolo de actuación de las universidades frente a situaciones de crisis humanitarias (2006), el Manifiesto de las universidades españolas en la campaña del milenio y en la lucha contra la pobreza (2006) y el documento Bases para la creación del Observatorio CUD (2007).

\subsection{Contribuciones de la Universidad en el sistema de Cooperación}

Gallart Parramón (2016: 2), en línea con la ESCUDE señala cuáles son las líneas esenciales en las que la Universidad puede contribuir en el ámbito de la cooperación:

- La formación y la educación.

- La Investigación para el desarrollo.

- Compartir experiencias compartiendo recursos.

- La incidencia en el entorno.

- La transferencia de tecnología. 
Lo que viene a señalar la ESCUDE es que «la investigación, la docencia y la transferencia de conocimiento serán los elementos que nos diferenciarán y aportarán valor añadido frente a otros actores del sistema de cooperación (ONGD, empresas, sindicatos, fundaciones, etc.)» (Gallart, 2016: 2).

Las universidades españolas participan en la elaboración, diseño, coordinación e implantación de las políticas de Cooperación al más alto nivel. Están representadas en el Consejo de Cooperación al Desarrollo estatal y forman parte activa del Observatorio de la Cooperación Española (OCUD) ${ }^{1}$. Asimismo, la CRUE cuenta con un activo Grupo de Trabajo de Cooperación en la sectorial CRUE Internacionalización y Cooperación que, entre otras actividades, informa anualmente al Ministerio de Asuntos Exteriores sobre las actividades y el desempeño realizado en este ámbito a través de una encuesta de seguimiento.

Estas funciones atribuidas a la Universidad se despliegan en un amplio abanico de actividades en el marco de la docencia, la investigación, la formación y sensibilización, y la firma de convenios internacionales entre universidades para la movilidad de toda la comunidad universitaria.

Así, OCUD señala las principales acciones, a modo de líneas maestras, que en materia de cooperación pueden desarrollar las universidades y que detallamos a continuación con nuestras aportaciones:

a. Acciones de formación, teórica y práctica, que son aquellas que llevan aparejada una carga crediticia y/o que están orientadas a obtener una titulación: Grados y posgrados vinculados al desarrollo y la cooperación, asignaturas, TFM y TFG ligados a los ODS, cursos de formación de formadores, etc. Formación específica de cooperantes y gestores de la cooperación al desarrollo. Se trata además de favorecer cambios en los planes de estudio para introducir asignaturas en 1 er y $2^{\circ}$ ciclo ligadas a la CUD, y llevar a cabo acciones conjuntas que mejoren la calidad de la formación no reglada.

b. Acciones de investigación, que comprenden tanto estudios sobre el desarrollo como para el desarrollo, a través de los distintos programas de doctorado, Cátedras o Institutos de Investigación, promoviendo la realización de tesis doctorales relacionadas con el ámbito de la cooperación. Son también aquellas acciones encaminadas a llevar a cabo proyectos de investigación conjuntos asociados al desarrollo sostenible y a la transferencia tecnológica adaptada a las condiciones locales.

c. Cooperación interuniversitaria, orientada al fortalecimiento de redes internacionales para la investigación conjunta en proyectos competitivos, la mejora de planes de estudio y la transferencia y difusión del conocimiento, capacidades y competencias en materia de desarrollo.

d. Acciones de difusión, sensibilización, movilización y educación para el desarrollo encaminadas a sensibilizar y dar a conocer a la comunidad universitaria los contenidos de los ODS, con el fin último de movilizar y comprometer a alumnado, profesorado y PAS en todos aquellos aspectos que refuercen la idea de ciudadanía solidaria y comprometida con los objetivos del horizonte 2030. Asimismo, son importantes las acciones de sensibilización coordinadas entre varias universidades orientadas a mostrar la importancia del desarrollo de los países con menos recursos y de las consecuencias del subdesarrollo.

e. Programas y Proyectos de acción sobre el terreno, en los que se desarrollen proyectos de investigación aplicada a la cooperación al desarrollo, incluyendo proyectos de consultoría y asistencia técnica a los distintos agentes de la cooperación que trabajan en ámbitos no universitarios, esto es, apoyar decididamente al tejido social de la cooperación.

f. Promoción, gestión y coordinación de políticas y programas CUD, con la celebración de congresos, jornadas, sesiones divulgativas, etc., en los que se analicen, coordinen y evalúen todos los aspectos relacionados con la gestión de la cooperación universitaria al desarrollo, y que contribuyan a la formación de expertos en desarrollo, especialmente entre los técnicos y gestores que van a trabajar en este ámbito. La visibilidad de estas acciones favorecerá la presencia activa de las universidades españolas en los órganos de decisión en materia de cooperación al desarrollo. Asimismo, se deben implementar programas propios en las universidades para financiar las acciones de cooperación, por ejemplo, la aplicación del concepto $0,7 \%$ en aportación fija al presupuesto o mediante aportaciones voluntarias de los distintos sectores de la comunidad universitaria, vía matrícula, vía sueldo de los trabajadores o mediante la captación de financiación externa de organismos nacionales e internacionales.

${ }^{1}$ Cfr. para una información más detallada la página web del OCUD: http://www.ocud.es/. 
En definitiva,elaborar una estrategia de Cooperación Universitaria al desarrollo en los centros educación superior supone concentrar esfuerzos, energías e iniciativas desde los órganos de gobierno, favorece la continuidad de las acciones y garantiza que se contemple desde su puesta en marcha el código de conducta para la CUD.

\subsection{El Código de conducta de las universidades para la Cooperación al Desarrollo}

El diseño y la implementación de las acciones que acabamos de puntear deberán implementarse bajo un código de conducta elaborado en 2015 y que pretende acotar el campo específico de la CUD, preservar su carácter solidario en sus objetivos y métodos de trabajo, y «enfatizar que su fin último debe ser el desarrollo sostenible del país con el que se coopera» (CRUE 2015: 2), en el entendido de que la cooperación internacional al desarrollo es un «proceso de progreso y cambio social, económico, político, cultural tecnológico, etc. que requiere organización participativa».

Entre los principios y objetivos de la CUD destaca la erradicación de la pobreza como su fin prioritario. Con este código se trata además de abrir a más y mejores oportunidades de vida digna de la ciudadanía, pero protegiendo de forma sostenible los recursos naturales y el acervo cultural de los pueblos. Otros objetivos señalados por este código de conducta para la CUD serán el fomento del acceso a los estudios universitarios, la equidad de género y trabajar por la paz, la democracia y el respeto a los derechos humanos.

La CUD ha de ser desinteresada y solidaria, con acciones coherentes a corto y largo plazo, y sin perder de vista que sus ámbitos naturales de actuación son la docencia y la investigación.

Se señala también cómo la universidad debe comprometerse institucionalmente implicando a toda su comunidad y favoreciendo el reconocimiento académico de las acciones de CUD.

En cuanto a las relaciones con las instituciones contraparte en otros países, el código destaca en su artículo 21 que la CUD debe entenderse como intercambio y enriquecimiento mutuo de las partes, al margen de imposiciones o actitudes paternalistas.

Por último, queremos destacar cómo el código incide en que las universidades deben favorecer el diálogo y los mecanismos de coordinación con el resto de agentes sociales de cooperación.

\section{LA UNIVERSIDAD Y LOS OBJETIVOS DE DESARROLLO SOSTENIBLE}

Los 17 Objetivos de Desarrollo Sostenible que Naciones Unidas pretende abordar y alcanzar en son los siguientes:

1. Acabar con la pobreza.

2. Hambre cero.

3. Salud y bienestar.

4. Educación de calidad.

5. Igualdad de género.

6. Agua limpia y saneamiento.

7. Energía asequible y no contaminante.

8. Trabajo decente y crecimiento económico.

9. Industria, innovación e infraestructura.

10. Reducción de las desigualdades.

11. Ciudades y comunidades sostenibles.

12. Producción y consumo responsables.

13. Acción por el clima.

14. Vida submarina.

15. Vida de ecosistemas terrestres.

16. Paz, justicia e instituciones solidarias

17. Alianzas para lograr los objetivos 
Cada ODS establece una serie de metas a cumplir para llegar al objetivo. Se trata, en definitiva, de «no dejar a nadie atrás» como señala Naciones Unidas. Para cada uno de ellos, el sistema universitario puede aportar en los planes de estudios de sus grados y posgrados un componente transversal de desarrollo sostenible. Bastaría con dotar a estos planes de estudio y guías docentes de contenidos y competencias que contribuyan a la adquisición de la sensibilidad y conciencia necesaria para alcanzar estos objetivos con éxito.

Por cuestiones de tiempo y espacio, y por ser el objeto de estudio de este encuentro, nos centraremos en esta comunicación en el ODS 4: Garantizar una educación inclusiva y equitativa de calidad y promover oportunidades de aprendizaje permanente para todos.

EI ODS 4 persigue el ambicioso objetivo de garantizar una educación inclusiva y equitativa de calidad y promover oportunidades de aprendizaje permanente para todos. Las Facultades de Educación de las universidades española siempre han sido sensibles a este objetivo, pero satisface ver cómo están cada vez más concienciadas con él y así se refleja en los planes de estudio de sus grados y posgrados en Educación. En este sentido es fundamental seguir desarrollando conocimiento, competencias e investigación que conduzcan a reforzar la educación de futuros docentes en la consecución del cumplimiento de los derechos humanos, el reconocimiento de la paz como valor fundamental de convivencia, el ejercicio responsable de la ciudadanía local y global, el desarrollo de políticas de igualdad de género, la consecución de un desarrollo realmente sostenible y la necesidad de una política sanitaria con vocación universal.

El Sistema Educativo Español se rige por los principios de calidad, cooperación, equidad, libertad de enseñanza, mérito, igualdad de oportunidades, no discriminación, eficiencia en la asignación de recursos públicos, transparencia y rendición de cuentas ${ }^{2}$. No obstante, aún queda mucho por conseguir. Se sigue observando en el sistema universitario español la brecha de género en las carreras técnicas o científicas. En la actualidad, y teniendo en cuenta que, según los datos del Ministerio de Educación y Formación Profesional (MEFP), las mujeres representan un 54,3 \% del alumnado matriculado y del $58,5 \%$ del total de egresados en estudios universitarios. Asimismo, la integración del alumnado con necesidades educativas especiales de cualquier índole ha de hacerse efectiva, así como la implantación progresiva de la nueva modalidad de Formación Profesional Dual que acerca la educación con mejor criterio al sector productivo.

Los contenidos educativos deben contemplar, a nuestro juicio, aspectos tanto cognitivos como no cognitivos del aprendizaje. Debe fomentarse asimismo la inclusión de competencias educativas para el desarrollo sostenible y la educación para la ciudadanía global, con capacidad crítica, que comprenda una educación intercultural, que abra los ojos de nuestro alumnado al mundo, preparando a niños, niñas y jóvenes para un mundo interconectado, pero desigual, y que necesita de ellos que entiendan la importancia de valores éticos como la igualdad, la justicia, la solidaridad y la sostenibilidad.

\section{CONCLUSIONES}

A lo largo de este trabajo hemos visto cómo la Agenda 2030 se define como un proceso participativo multinivel y multiactor y, por lo tanto, su implementación y desarrollo debe seguir esta misma dinámica de trabajo que la convierta en una realidad de éxito. Para ello se necesita el trabajo y el compromiso de todos los actores, y la universidad puede ser su motor, haciendo lo que mejor sabe hacer: formar y educar ciudadanos para conseguir un mundo mejor desde el conocimiento y la investigación.

La generación de conocimiento en la universidad y su difusión se ponen al servicio de la aldea global con impulso decidido, acometiendo con la vista puesta en los ODS y el horizonte 2030 las acciones necesarias para producir cambios estructurales en beneficio de toda la sociedad, modificando o erradicando estructuras ineficientes, erróneas o injustas.

La estrategia para un desarrollo sostenible ha de ser parte principal en la hoja de ruta de las universidades españolas. La academia debe contribuir a afrontar los retos y necesidades a los que 
se enfrenta la ciudadanía del siglo XXI. La política universitaria, junto con las políticas nacionales e internacionales han de ser coherentes y tener como fin último conseguir una sociedad inclusiva y responsable que transforme el mundo. En definitiva, la universidad debe formar para transformar, y hacerlo cada vez mejor.

La misión de las universidades, tanto públicas como privadas, ni puede ni debe agotarse con la formación de profesionales altamente cualificados. El ámbito de la educación superior ha de ser también un espacio privilegiado para la formación en valores solidarios, un espacio para el diálogo, el debate y una mejor formación ciudadana. Solo así será capaz de contribuir con eficacia al cumplimiento de los ODS en el horizonte 2030.

\section{REFERENCIAS BIBLIOGRÁFICAS}

CEURI-CRUE (2000). Estrategia de las Universidades españolas en materia de Cooperación al Desarrollo (ESCUDE). Córdoba: CRUE.

CRUE (2001). Universidad: Compromiso social y voluntariado. Madrid: CRUE.

CRUE (2006). Manifiesto de las universidades españolas en la campaña del milenio y en la lucha contra la pobreza. Madrid: CRUE.

CRUE (2006). Protocolo de actuación de las universidades frente a situaciones de crisis humanitarias. Girona: CRUE.

CRUE (2015). Código de conducta de las universidades en materia de cooperación internacional. Madrid: CRUE.

CRUE-AECI (2007). Bases para la creación del Observatorio. Madrid: CRUE.

Gallart Parramon, S. (2016) «Introducción: La Cooperación Universitaria al desarrollo en la Universidad Carlos III de Madrid». En Pedraza Córdoba, J. Cooperación Universitaria para la educación (1-11). Granada: Comares. 